Memoirs of the College of Science, University of Kyoto, Series A

Vol. XXXII, Mathematics No. 3, 1960.

\title{
Quelques remarques sur la structure projective d'espace fibré
}

\author{
Par \\ Jōyō Kanitani, Seizi Takizawa
}

(Reçu le 21 Décembre, 1959)

Dans un mémoire précédent [1] nous avons démontré qu'il existe, au dessus d'une variété différentiable paracompacte à deux dimensions, un champ de tenseurs non-triviaux covariants symétriques du troisième ordre qui est apolaire au champ de tenseurs fondamentaux (covariants symétriques définis positifs du second ordre). Nous démontrerons, dans cet article, ce fait pour la variété différentiable paracompacte $M$ à $n$ dimensions, et d'ailleurs que nous pouvons choisir un tel champ de la manière qi'il ne s'annule pas dans la variété $M$ toute entière, et ensuite que, si $n=2$ et si la variété $M$ est compacte, il existe un tel champ qui n'admet que des points singulier de nombre fini.

En prenant ensuite le cas où $n=3$, nous introduisons une structure géométrique relative la variété $M$, qui peut être regardée come une généralization de la directrice de Wilczynski d'une surface dans un espace projectif.

1. Soit $M$ une variété différentiable paracompacte à $n$ dimensions. Prenons un point $x \in M$. Soient $U \ni x$ le domaine d'un système de coordonnées locales $u^{1}, \cdots, u^{n}, T^{*}$ l'espace de covecteurs tangents à $M$ en $x, \sigma$ le champ de tenseurs fondamentaux. Supposons que ce champ s'exprime, dans $U$, par

$$
\sigma=a_{i j}(x) d u^{i} \otimes d u^{j} .
$$

Soit $Y_{x}=\left\{y_{i j k} d u^{i} \otimes d u^{j} \otimes d u^{k}\right\}$ l'espace vectoriel formé par les éléments symétriques de $T_{x}^{*} \otimes T_{x}^{*} \otimes T_{x}^{*}$. Envisageons l'application linéaire $\varphi: Y_{x} \rightarrow T_{x}^{*}$ définié par

$$
\varphi\left(y_{i j k} d u^{i} \otimes d u^{j} \otimes d u^{k}\right)=y_{l_{m i}} a^{l m} d u^{i} \quad\left(\left(a^{j i}\right)=\left(a_{i j}\right)^{-1}\right) .
$$


Etant donné un covecteur $v_{i} d u^{i} \in T_{x}^{*}$, si l'on pose

$$
y_{i j k}=\frac{1}{n+2}\left(v_{i} a_{j k}+v_{j} a_{i k}+v_{k} a_{i j}\right),
$$

il vient $y_{s t i} a^{s t}=v_{i}$. C'est-à-dire que l'application $\phi$ est une surjection. Ainsi, $W_{x}=\operatorname{ker} \rho$ est un espace vectoriel tel que

$$
\operatorname{dim} Y_{x}-\operatorname{dim} W_{x}=\operatorname{dim} T_{x}^{*},
$$

ou bien

$$
N=\operatorname{dim} W_{x}=\left(\begin{array}{l}
n \\
3
\end{array}\right)+n^{2}-n .
$$

2. Si l'on pose

$$
\begin{gathered}
\theta^{i j k}=S d u^{i} \otimes d u^{j} \otimes d u^{k} \quad(S: \text { symétrisation }) \\
\tilde{\theta}^{i j k}=\theta^{i j k}-\frac{1}{3}\left(a^{i j} \delta_{1}^{k}+a^{j k} \delta_{1}^{i}+a^{i k} \delta_{1}^{j}\right) \frac{\theta^{111}}{a^{11}} \\
\ldots \ldots \ldots \ldots \ldots \ldots \ldots \ldots \ldots \ldots \ldots \ldots \\
- \\
-\frac{1}{3}\left(a^{i j} \delta_{n}^{k}+a^{j k} \delta_{n}^{i}+a^{i k} \delta_{n}^{j}\right) \frac{\theta^{n n n}}{a^{n n}}
\end{gathered}
$$

il vient

$$
\tilde{\theta}^{111}=0, \tilde{\theta}^{222}=0, \cdots, \tilde{\theta}^{n n n}=0,
$$

et les autres $\tilde{\theta}^{i j k}$ que nous désignerons par $\tilde{\theta}^{i^{\prime} j^{\prime} k^{\prime}}$ se font un système de base de l'espace vectoriel $W_{x}$.

Si $x \in U \cap U^{\prime}$, on a

$$
\begin{gathered}
\sum W_{i^{\prime} j^{\prime} k^{\prime}} \tilde{\theta}^{i^{\prime} j^{\prime} k^{\prime}}=W_{i j k} \theta^{i j k} \quad\left(a^{i j} W_{i j k}=0\right) \\
=W_{i j k} Q_{r}^{i} Q_{s}^{j} Q_{t}^{k} \theta^{\prime r s t}=\sum W_{i j k} Q_{r}^{i} Q_{s^{\prime}}^{\prime} Q_{t^{\prime}}^{k} \tilde{\theta}^{\prime r^{\prime} s^{\prime} t^{\prime}} \\
\left(Q_{j}^{i}=\frac{\partial u^{i}}{\partial u^{\prime j}}\right) .
\end{gathered}
$$

Nous pouvons donc définir une représentation linéaire $\mathscr{R}$ du groupe linéaire $L_{n}=\left(Q_{j}^{i}\right)$ sur l'espace vectoriel $W_{x}$ par

$$
\begin{aligned}
& W_{r^{\prime} s^{\prime} t^{\prime}}^{\prime}=\sum\left[Q_{r^{\prime}}^{i^{\prime}} Q_{s^{\prime}}^{j^{\prime}} Q_{t^{\prime}}^{k^{\prime}}-\frac{1}{3}\left(a^{i^{\prime} j^{\prime}} \delta_{1}^{k^{\prime}}+a^{j^{\prime} k^{\prime}} \delta_{1}^{i^{\prime}}+a^{i^{\prime} k^{\prime}} \delta_{1}^{j^{\prime}}\right) \frac{Q_{r}^{1} Q_{s}^{1} Q_{t^{\prime}}^{1}}{a^{11}}\right. \\
& \left.-\frac{1}{3}\left(a^{i^{\prime} j^{\prime}} \delta_{n}^{k^{\prime}}+a^{j^{\prime} k^{\prime}} \delta_{n}^{i^{\prime}}+a^{i^{\prime} k^{\prime}} \delta_{n}^{j^{\prime}}\right) \frac{Q_{r}^{n} Q_{\varepsilon^{n}}^{n} Q_{t^{\prime}}^{n}}{a^{n n}}\right] W_{i^{\prime} j^{\prime} k^{\prime}} .
\end{aligned}
$$


Nous pouvons donner à la réunion

$$
E=\bigcup_{x \in M} W_{x}
$$

une structure d'espace fibré, de base $M$, de fibre-type un espace vectoriel $W$ à $N$ dimensions, de groupe structural $L_{n}$ dont l'opération sur $W$ se fait la représentation linéaire $R$.

Une section locale arbitrairement donnée de $E\left(M, W, L_{n}\right)$ au dessus d'un voisinage peut être étendue pour la variété $M$ toute entiére ([2], p. 54). La section ainsi obtenue s'exprime, dans $U$, par

$$
\sum \tilde{b}_{i^{\prime} j^{\prime} k^{\prime}}(x) \tilde{\theta}^{i^{\prime} j^{\prime} k^{\prime}}=b_{i j k} \theta^{i j_{k}} \quad\left(a^{s t} b_{s t i}=0\right) .
$$

Nous voyons ainsi qu'il existe un champ $\tau$ de tenseurs covariants symétriques non-triviaux du troisiéme ordre, qui est apolaire au champ $\sigma$ de tenseurs fondamentaux.

3. Nous allons maintenant démontrer que nous pouvons choisir le champ de tenseurs $\tau$ de telle sorte qu'il ne s'annule pas dans $M$. Soit $Z_{x}$ l'ensemble des éléments symétriques définis positifs de $W_{x}^{*} \otimes W_{x}^{*}$. Envisageons la section de l'espace fibré qui s'obtient par la donnation à la réunion

$$
\bigcup_{x \in M} Z_{x}
$$

une structure d'espace fibré de la même maniére que nous avons introduit le champ de tenseurs fondamentaux $\sigma$. Portons dans $W_{x}$ la métrique définie au moyen de cette section et considérons $(N-1)$ sphére

$$
S_{x}=\left\{y \in W_{x}, \text { longueur }|y|=1\right\} .
$$

Donnons à la réunion

$$
B=\bigcup_{x \in M} S_{x}
$$

la structure d'espace fibré $B\left(M, S^{N-1}, O_{N}\right)$ où $O_{N}$ est le groupe orthogonal. La recherche du champ de tenseurs $\tau$ qui ne s'annule pas dans $M$ se raméne à celle de la section de $B\left(M, S^{N-1}, O_{N}\right)$. L'existence de la section derniére est bien assurée, car nous avons, si $n \geq 3$,

$$
N-1=\left(\begin{array}{l}
n \\
3
\end{array}\right)+n^{2}-n-1 \geq n=\operatorname{dim} M
$$


ce qui nous montre que ([2], p. 148)

$$
\pi_{i}\left(S^{N-1}\right)=0 \quad(i=1, \cdots, N-2) .
$$

Lorsque $n=2$ on a $N=n$, à savoir, $W_{x}$ est isomorphe à $T_{x}^{*}$. Nous pouvons donc choisir, si la variété $M$ est compacte, le champ de covecteurs $\tau$ de telle sorte qu'il n'admette que des points singulière de nombre fini ([3] p. 548). Cela revient à dire que

$$
\theta=a^{i r} a^{j s} a^{k t} b_{i j k} b_{r s t}
$$

ne s'annule qu'à points de nombre fini ([1] p. 83).

4. En faisant l'usage des notations introduites dans [1], posons

$$
\begin{aligned}
k_{l m i j}= & K_{l i s} K_{m j}^{s}-K_{l j s} K_{m i}^{s} \\
+ & \frac{1}{n-2}\left(H_{l_{i}} K_{? n j}^{s} K_{j_{s}}^{t}-H_{m i} K_{l t}^{s} K_{j_{s}}^{t}\right. \\
& \left.-H_{l j} K_{m t}^{s} K_{i s}^{t}+H_{m j} K_{l t}^{s} K_{i s}^{t}\right) \\
& -\frac{1}{(n-1)(n-2)}\left(H_{l i} H_{m j}-H_{l j} H_{m i}\right) K_{t}^{r s} K_{r s}^{t} .
\end{aligned}
$$

Il vient

$$
\begin{gathered}
k_{m l i j}=-k_{l_{m i j}}, \quad k_{l_{m j i}}=-k_{l_{m i j}}, \quad k_{i j l m}=k_{l m i j}, \\
H^{m j} k_{l_{m i j}}=0 .
\end{gathered}
$$

Lorsque $n=3$, en y faisant $l=1, i=1,2,3$, nous avons

$$
\begin{aligned}
& H^{22} k_{1212}+2 H^{23} k_{1213}+H^{33} k_{1313}=0, \\
& H^{21} k_{1221}+H^{31} k_{1321}+H^{23} k_{1223}+H^{33} k_{1323}=0, \\
& H^{21} k_{1231}+H^{31} k_{1331}+H^{22} k_{1232}+H^{32} k_{1332}=0 .
\end{aligned}
$$

Ajoutons ces équations multipliées respectivement par $H^{i 1}, H^{i 2}$, $H^{i s}(i=1,2,3)$. Nous obtenons

$$
\begin{aligned}
& H_{21} k_{3123}+H_{22} k_{3131}+H_{23} k_{3112} \\
& \quad+H_{31} k_{1223}+H_{32} k_{1231}+H_{33} k_{1212}=0, \\
& H_{11} k_{3123}+H_{12} k_{3131}+H_{13} k_{3112}=0, \\
& H_{11} k_{1223}+H_{12} k_{1231}+H_{13} k_{1212}=0 .
\end{aligned}
$$

De même, en faisant $l=2,3$ dans (1), on tire

$$
\begin{aligned}
& H_{31} k_{1223}+H_{32} k_{1231}+H_{33} k_{1212} \\
& \quad+H_{11} k_{2323}+H_{12} k_{2331}+H_{13} k_{2312}=0,
\end{aligned}
$$


Quelques remarques sur la structure projective d'espace fibré 449

$$
\begin{aligned}
& H_{21} k_{1223}+H_{22} k_{1231}+H_{23} k_{1212}=0, \\
& H_{21} k_{2323}+H_{22} k_{2331}+H_{23} k_{2312}=0, \\
& H_{11} k_{2333}+H_{12} k_{2331}+H_{13} k_{2312} \\
& \quad+H_{21} k_{3123}+H_{22} k_{3131}+H_{23} k_{3112}=0, \\
& H_{31} k_{2323}+H_{32} k_{2331}+H_{33} k_{2312}=0, \\
& H_{31} k_{3123}+H_{32} k_{3131}+H_{33} k_{3112}=0 .
\end{aligned}
$$

En résumé nous avons

$$
H_{i 1} k_{l_{m 23}}+H_{i 2} k_{l_{m 31}}+H_{i 3} k_{l_{m 12}}=0 \quad(i, l, m=1,2,3)
$$

et, par suite,

$$
k_{l m i j}=0 \quad(l, m, i, j=1,2,3) .
$$

Ensuite, si l'on pose

$$
\begin{aligned}
\langle l m i j\rangle= & \{l m i j\} \\
- & \frac{1}{n-2}\left(a_{l i}\left\{\begin{array}{c}
s \\
m \cdot j s
\end{array}\right\}-a_{m i}\left\{\begin{array}{l}
s \\
l \cdot j s
\end{array}\right\}\right. \\
& \left.-a_{l j}\left\{\begin{array}{c}
s \\
m \cdot i s
\end{array}\right\}+a_{m j}\left\{\begin{array}{l}
s \\
l \cdot i s
\end{array}\right\}\right) \\
+ & \frac{1}{(n-1)(n-2)}\left(a_{l m} a_{m j}-a_{l j} a_{m i}\right)\left\{\begin{array}{l}
\{t \\
\cdot \cdot s_{t}
\end{array}\right\},
\end{aligned}
$$

il vient aussi

$$
\begin{aligned}
& \langle m l i j\rangle=-\langle l m i j\rangle, \quad\langle l m j i\rangle=-\langle l m i j\rangle, \quad\langle i j l m\rangle=\langle l m i j\rangle, \\
& \quad a^{m j}\langle l m i j\rangle=0 .
\end{aligned}
$$

Nous avons donc, lorsque $n=3$,

$$
\langle l m i j\rangle=0 \quad(l, m, i, j=1,2,3) .
$$

Or, nous avons d'après $(3.4),(4.2)_{n},(5.3)$ de [1]

$$
R_{h k i j}-R_{k h i j}=\frac{1}{\kappa}\langle h k i j\rangle-k_{h k i j} .
$$

Nous obtenons ainsi, lorsque $n=3$,

$$
R_{h k i j}=R_{k h i j} \quad(h, k, i, j=1,2,3) .
$$

5. Supposons que $n=3$. L'équation $H^{k j}\left(R_{k k i j}+R_{k h i j}\right)=0$ devient alors d'après (4.2)

$$
H^{k j} R_{h k i j}=0
$$


Posons

$$
\boldsymbol{K}_{i j l}=H_{l 1} R_{i j 23}+H_{l 2} R_{i j 31}+H_{l 3} R_{i j 12} .
$$

de sorte qu'on a

$$
\boldsymbol{K}_{i j l}=\boldsymbol{K}_{j i l} .
$$

L'équation (5.1) peut s'écrire

$$
\boldsymbol{K}_{i j l}=\boldsymbol{K}_{i l j} \text {. }
$$

D'ailleurs nous avons d'après (5.2) de [1] (p. 163)

$$
H^{h_{k}} \boldsymbol{K}_{h k l}=0 \text {. }
$$

Nous appellerons donc la forme $\boldsymbol{K}_{i j l} d u^{i} d u^{j} d u^{l}$ la forme de Darboux induite. Il suit de (5.2)

$$
R_{i j 23}=\boldsymbol{K}_{i j}^{1}, \quad R_{i j 31}=\boldsymbol{K}_{i j}^{2}, \quad R_{i j 12}=\boldsymbol{K}_{i j}^{3}
$$

Posons

$$
P_{h i j}=R_{h i j}^{0}-H_{h m} R_{n+1 \cdot i j}^{m} .
$$

Nous avons ((6.3) de [1])

$$
H^{h j} P_{h i j}=0
$$

ce qui nous donne

$$
H_{h 1} P_{k 23}+H_{h 2} P_{k 31}+H_{h 3} P_{k 12}=H_{k 1} P_{h 23}+H_{k 2} P_{h 31}+H_{k 3} P_{h 12} .
$$

Grâce à (2.6) de [1] (l'identité de Bianchi), nous avons

$$
\begin{gathered}
\left(\frac{\partial R_{h k i j}}{\partial u^{l}}-\Pi_{h l}^{s} R_{s k i j}-\Pi_{k l}^{s} R_{h s i j}+H_{k l} R_{h i j}^{0}-H_{h l} R_{n+1 k i j}\right. \\
\left.-\frac{1}{2} K_{k l}^{s} R_{h s i j}+\frac{1}{2} K_{h l}^{s} R_{s k i j}\right) d u^{l} \wedge d u^{i} \wedge d u^{j}=0 \\
(h, k, i, j=1,2,3) .
\end{gathered}
$$

Ajoutons membre à membre à cette équation ce qu'on obtient en y échangeant $h, k$. Nous obtenons alors grâce aux (4.2), (5.3), (5. 4)

$$
\begin{gathered}
\boldsymbol{K}_{h k ; s}^{s}+H_{h 1} P_{k 23}+H_{h 2} P_{k 31}+H_{h 3} P_{k 12}=0 \\
(h, k=1,2,3)
\end{gathered}
$$

et, par suite,

(5. 5) $\quad P_{k 23}=-\boldsymbol{K}_{k ; s}^{1 s}, \quad P_{k 31}=-\boldsymbol{K}_{k ; s}^{2 s}, \quad P_{k 12}=-\boldsymbol{K}_{k ; s}^{3 s}$. 
Quelques remarques sur la structure projective d'espace fibré 451

En retranchant l'une de l'autre les deux équations que nous venons de mentionner nous obtenons

$$
K_{h t}^{s} \boldsymbol{K}_{k s}^{t}=K_{k t}^{s} \boldsymbol{K}_{h s}^{t} .
$$

6. Soient $X=t^{s} \Lambda_{s}, X^{\prime}=t^{\prime s} \Lambda_{s}$ les vecteurs horizontaux ([1], p. 158). Posons

$$
v_{1}=\left|\begin{array}{cc}
t^{2} & t^{3} \\
t^{\prime 2} & t^{\prime 3}
\end{array}\right|, \quad v_{2}=\left|\begin{array}{cc}
t^{3} & t^{1} \\
t^{\prime 3} & t^{\prime \prime}
\end{array}\right|, \quad v_{3}=\left|\begin{array}{cc}
t^{1} & t^{2} \\
t^{1 /} & t^{\prime 2}
\end{array}\right|
$$

La sous-algèbre d'holonomie de Lie-algèbre du groupe structural $P$ est engendrée par les éléments

$$
\Omega_{\lambda}^{\mu}=q_{\beta}^{\mu} p_{\lambda}^{\alpha} R_{\alpha_{i j}}^{\beta} t^{i} t^{\prime j} .
$$

Puisque

$$
\begin{gathered}
R_{0 i j}^{\beta}=R_{\alpha i j}^{n+1}=0, \quad p_{0}^{K}=q_{0}^{K}=0, \quad p_{A}^{n+1}=q_{A}^{n+1}=0 \\
(\alpha, \beta=0,1, \cdots, n ; K=1, \cdots, n+1 ; A=0, \cdots, n),
\end{gathered}
$$

nous avons

$$
\begin{gathered}
\Omega_{0}^{\beta}=0, \quad \Omega_{\alpha}^{n+1}=0, \\
\Omega_{h}^{k}=q_{m}^{k} p_{h}^{l} R_{l i j}^{m} t^{i} t^{\prime}=\frac{1}{2} q_{m}^{k} p_{h}^{l} K_{l}^{m s} v_{s} \\
(h, k=1,2,3 ; l, m: 1 \rightarrow 3) .
\end{gathered}
$$

Envisageons l'homographique représentant un tel élément:

$$
\left\{\begin{array}{l}
\lambda \xi^{\prime 0}=\Omega_{i}^{0} \xi^{i}+\Omega_{4}^{0} \xi^{4}, \\
\lambda \xi^{\prime k}=\Omega_{i}^{k} \xi^{i}+\Omega_{4}^{k} \xi^{4} \quad(k=1,2,3), \\
\lambda \xi^{\prime 4}=0 .
\end{array}\right.
$$

Considérons dans $\boldsymbol{S}_{4}$ un hyperplan $u_{\alpha}$. Son pôle par rapport à la quadrique

$$
2 \xi^{0} \xi^{4}=H_{i j} \xi^{i} \xi^{j} \quad(i, j: 1 \rightarrow 3)
$$

que nous appllerons la quadrique de Lie est donné par

$$
\xi^{0}=-u_{4}, \quad \xi^{i}=H^{i j} u_{j}, \quad \xi^{4}=-u_{0} .
$$

Le portant dans $(6.1)$ nous obtenons

$$
\begin{aligned}
\lambda \xi^{\prime 0} & =\Omega^{0 s} u_{s}-\Omega_{4}^{0} u_{0}, \\
\lambda \xi^{\prime k} & =\Omega^{k s} u_{s}-\Omega_{4}^{k} u_{0}, \\
\lambda \xi^{\prime 4} & =0
\end{aligned}
$$

ce qui détermine une correlation projective dans l'espace $o=A A_{1} A_{2} A_{3}$ 
amenant le plan $\left(u_{0}, u_{1}, u_{2}, u_{3}\right)(=u \cap o)$ au point $\left(\xi^{\prime 0}, \xi^{\prime 1}, \xi^{\prime 2}, \xi^{\prime 3}\right)$. Le lieu des éléments d'incidence dans cette correlation est la quadrique

$$
\Omega^{i j} u_{i} u_{j}+\left(\Omega^{0 i}-\Omega_{4}^{i}\right) u_{0} u_{i}-\Omega_{4}^{0}\left(u_{0}\right)^{2}=0 .
$$

Le pôle du plan $\left(\delta_{0}^{k}, \delta_{1}^{k}, \delta_{2}^{k}, \delta_{3}^{k}\right)(k=1,2,3)$ par rapport à cette quadrique est le point donné par

$$
\Omega^{k i} u_{i}+\frac{1}{2}\left(\Omega^{0 k}-\Omega_{4}^{k}\right) u_{0}=0 .
$$

Ainsi, le plan polaire $\pi$ du point $A$ (l'intersection des trois plans considérés) passe par les trois points

$$
\left(\frac{1}{2}\left(\Omega_{k}^{0}-\Omega_{4 k}\right), \Omega_{k}^{1}, \Omega_{k}^{2}, \Omega_{k}^{3}\right) \quad(k=1,2,3)
$$

qui déterminent ce plan polaire $\pi$ pourvu que la quadrique (6.2) soit régulière. Si l'on prend comme point de départ du chemin horizontal le point $\left(u^{i}, \delta_{a}^{\beta}\right)$, les points $(6.3)$ deviennent

$$
\left(-\frac{1}{2} \boldsymbol{K}_{k ; s}^{s t} v_{t}, \boldsymbol{K}_{k}^{1 t} v_{t}, \boldsymbol{K}_{k}^{2 t} v_{t}, \boldsymbol{K}_{k}^{3 t} v_{t}\right)
$$

Or, en considérant le cas où $n=2$, si l'on y remplace $\boldsymbol{K}_{k}^{i j}$ par $K_{k}^{i j}(i, j, k=1,2)$ on obtient les expressions de duex points dont la droite de jonction est la directrice de Wilczynski ([4], p. 203).

Nous pouvons donc regarder le plan polaire $\pi$ comme directrice de Wilczynski généralisée de $M$.

Les hyperplans homologues dans la transformation projective (6. 1) se relient au moyen des équations

$$
\left\{\begin{array}{l}
\sigma u_{0}^{\prime}=0, \\
\sigma u_{i}^{\prime}=\Omega_{i}^{0} u_{0}+\Omega_{i}^{k} u_{k}, \\
\sigma u_{4}^{\prime}=\Omega_{4}^{0} u_{0}+\Omega_{4}^{k} u_{k} .
\end{array}\right.
$$

L'hyperplan polaire d'un point $\xi$ par rapport à la quadrique de Lie est donné par

$$
u_{0}=-\xi^{4}, \quad u_{i}=H_{i j} \xi^{j}, \quad u_{4}=-\xi^{0} .
$$

En effectuant, ensuite, la transformation (6.4) nous obtenons la correlation projective

$$
\left\{\begin{array}{l}
\sigma u_{0}^{\prime}=0 \\
\sigma u_{i}^{\prime}=\Omega_{i j} \xi^{j}-\Omega_{i}^{0} \xi^{4} \\
\sigma u_{4}^{\prime}=\Omega_{4 j} \xi^{j}-\Omega_{4}^{0} \xi^{4}
\end{array}\right.
$$


dans laquelle le lieu des éléments d'incidence est le cône

$$
\Omega_{i j} \xi^{i} \xi^{j}-\left(\Omega_{i}^{0}-\Omega_{4 i}\right) \xi^{i} \xi^{4}-\Omega_{4}^{0}\left(\xi^{0}\right)^{2}=0 .
$$

La droite conjuguée à l'hyperplan $o=A A_{1} A_{2} A_{3}$ par rapport à ce cône se trouve dans les trois hyperplans

$$
\left(0, \Omega_{k 1}, \Omega_{k 2}, \Omega_{k 3},-\frac{1}{2}\left(\Omega_{k}^{0}-\Omega_{4 k}\right)\right) \quad(k=1,2,3) .
$$

qui sont les hyperplans polaires des points (6.3) par rapport à la quadrique de Lie.

\section{RÉFÉRENCES}

[1] J. Kanitani, Sur le faisceau canonique d'une variété différentiable (Mem. Coll. Sci. Univ. Kyoto, Ser. Math. Vol. 32, 1959).

[2] N. Steenrod, The topology of fibre bundles.

[3] P. Alexandroff, H. Hopf, Topologie.

[4] G. Fubini, E. Chech, Introduction à la géométrie projective différentielle des surfaces. 